\title{
A CHARACTERIZATION THEOREM FOR MONOTONE MAPPINGS
}

\author{
E. E. FLOYD AND M. K. FORT, JR.
}

1. Introduction. Our main result states that a mapping (i.e. continuous function) $f$ on the 2 -sphere $S$ onto itself is monotone if and only if $f$ has a continuous extension $g$ which maps the interior of $S$ homeomorphically onto itself.

The fact that the above condition is sufficient for $f$ to be monotone has been proved by Floyd in [1]. The necessity will follow from results obtained by Fort in [2] and Youngs in [3].

2. Notation and terminology. If $X$ is a topological space, $H(X)$ is the group of all homeomorphisms of $X$ onto $X$. We topologize $H(X)$ with the compact-open topology.

We let $P$ be a plane, and let $S$ be the unit 2-sphere in Euclidean 3-space. Both $H(P)$ and $H(S)$ are topological groups (under the compact open topology). It has been proved in [2] that $H(P)$ is locally arcwise connected.

By a curve in a topological space $Y$ we mean a continuous function on $[0,1]$ into $Y$. If $\Phi$ is a curve and $0 \leqq t \leqq 1$, we denote the value of $\Phi$ at $t$ by $\Phi_{t}$. A curve $\Phi$ joins $x$ to $y$ in a set $K$ if $\Phi_{0}=x, \Phi_{1}=y$, and $\Phi_{t} \in K$ for all $t$ in $[0,1]$. A curve $\Phi$ in a metric space has diameter less than $\epsilon$ if and only if the distance from $\Phi_{t}$ to $\Phi_{\varepsilon}$ is less than $\epsilon$ for all $t, s$ in $[0,1]$.

We define $R(S)$ to be the set of all rotations of $S$, and we let $I$ be the identity mapping of $S$ onto $S$. We let $p=(0,0,1)$ and then define $F(S)$ to be the set of all functions $f \in H(S)$ for which $f(p)=p$. Both $R(S)$ and $F(S)$ are obviously subgroups of $H(S)$.

We let $d$ be the usual metric for $S$, and for mappings $f, g$ of $S$ onto $S$ we define $\rho(f, g)=\sup _{x \in S} d(f(x), g(x))$. It is well known that the topology induced on $H(S)$ by $\rho$ is the compact-open topology, and hence $\rho$ is an admissible metric for $H(S)$.

\section{A local connectedness theorem.}

ThEOREM 1. $H(S)$ is locally arcwise connected.

Proof. It is obvious that $R(S)$ is locally arcwise connected. We now prove that $F(S)$ is also locally arcwise connected. This is accomplished by exhibiting a homeomorphism from $F(S)$ onto $H(P)$.

Presented to the Society, April 25, 1953; received by the editors March 11, 1953. 
We let $\pi$ be the stereographic projection from $p$ of $S-\{p\}$ onto $P$. If $f \in F(S)$, we define $T(f)=\pi f \pi^{-1}$. It is easy to verify that $T$ is a homeomorphism of $F(S)$ onto $H(P)$. Since $H(P)$ is locally arcwise connected, it follows that $F(S)$ is also locally arcwise connected.

In order to prove that $H(S)$ is locally arcwise connected, it is sufficient to prove that corresponding to each $\epsilon>0$ there exists a $\delta>0$ such that if $f \in H(S)$ and $\rho(f, I)<\delta$ then $f$ and $I$ may be joined in $H(S)$ by a curve of diameter less than $\epsilon$.

Suppose $\epsilon>0$. There exists $\alpha>0$ such that if $\phi$ and $\psi$ are members of $H(S)$ which are in the $\alpha$-neighborhood of $I$, then $\phi \psi$ is in the $\epsilon / 2$-neighborhood of $I$. There exists $\beta>0$ such that: if $g \in R(S)$ and $\rho(g, I)<\beta$, then $g$ and $I$ can be joined in $R(S)$ by a curve of diameter less than $\alpha$; and if $h \in F(S)$ and $\rho(h, I)<\beta$, then $h$ and $I$ can be joined in $F(S)$ by a curve of diameter less than $\alpha$. We define $\delta=\beta / 2$.

Suppose $f \in H(S)$ and $\rho(f, I)<\delta$. There exists $g \in R(S)$ such that $\rho(g, I)<\delta$ and $g f(p)=p$. Hence $g f \in F(S)$. It is easily seen that $\rho(g f, I)<2 \delta=\beta$, and that $\rho\left(g^{-1}, I\right)<\beta$. Therefore there exists a curve $\Phi$ of diameter less than $\alpha$ which joins $I$ to $g^{-1}$ in $R(S)$, and there exists a curve $\Psi$ of diameter less than $\alpha$ which joins $I$ to $g f$ in $F(S)$. We define a curve $\Omega$ by letting $\Omega_{t}=\Phi_{t} \Psi_{t}$ for $0 \leqq t \leqq 1$. It is easily seen that $\Omega$ is a curve of diameter less than $\epsilon$ which joins $I$ to $f$ in $H(S)$. Thus $H(S)$ is locally arcwise connected at $I$ and, since $H(S)$ is a topological group, this implies that $H(S)$ is locally arcwise connected.

CoROLlaRy 1. $H(S)$ is uniformly locally arcwise connected with respect to the metric $\rho$.

Proof. We make use of the fact that $\rho(f, g)=\rho(f h, g h)$ for all $f, g$, and $h$ in $H(S)$.

Suppose $\epsilon>0$. There exists $\delta>0$ such that if $h \in H(S)$ and $\rho(h, I)<\delta$ then $h$ can be joined to $I$ in $H(S)$ by a curve of diameter less than $\epsilon$.

Suppose $f$ and $g$ are in $H(S)$ and $\rho(f, g)<\delta$. Then $\rho\left(f g^{-1}, I\right)<\delta$. There exists a curve $\Phi$ of diameter less than $\epsilon$ which joins $I$ to $f g^{-1}$ in $H(S)$. We define a curve $\Psi$ by letting $\Psi_{t}=\Phi_{t} g$ for $0 \leqq t \leqq 1$. Then $\Psi$ is a curve of diameter less than $\epsilon$ which joins $g$ to $f$ in $H(S)$. It follows from the arcwise connectedness theorem that there exists an arc of diameter less than $\epsilon$ which joins $g$ to $f$ in $H(S)$. Thus $H(S)$ is uniformly locally arcwise connected.

We now let $M(S)$ be the set of all monotone mappings of $S$ onto $S$, and metrize $M(S)$ by $\rho$.

CoRollary 2. $M(S)$ is uniformly locally arcwise connected with respect to the metric $\rho$. 
Proof. Youngs has proved in [3] that $H(S)$ is dense in $M(S)$. Our result therefore follows readily from Corollary 1.

4. A characterization of monotone mappings of $S$ onto $S$. We let $Q$ be the interior of $S$.

Theorem 2. $A$ mapping $f$ of $S$ onto $S$ is monotone if and only if there exists a continuous extension $g$ of $f$ such that $g$ maps $S \cup Q$ onto itself and $g \mid Q$ is a homeomorphism of $Q$ onto $Q$.

Proof. It follows immediately from a theorem due to Floyd (see $[1$, p. 228]) that if such a $g$ exists, then $f$ is monotone.

Now suppose that $f$ is monotone. We consider the space $M(S)$ metrized by $\rho$. Young's approximation theorem states that $f$ is a limit point of $H(S)$. Since $H(S)$ is uniformly locally arcwise connected, it follows that there exists an arc $\Phi$ such that $\Phi_{1}=f$ and $\Phi_{t} \in H(S)$ for $0 \leqq t<1$. Each point of $S \cup Q$ can be represented in the form $t x$ where $x \in S$ and $0 \leqq t \leqq 1$. We define $g(t x)=t \Phi_{t}(x)$ for all $x \in S$ and $0 \leqq t \leqq 1$. It is easily seen that $g$ is a continuous function on $S \cup Q$ onto $S \cup Q$, that $g \mid S=f$, and that $g \mid Q$ is a homeomorphism of $Q$ onto $Q$.

\section{BIBLIOGRAPHY}

1. E. E. Floyd, The extension of homeomorphisms, Duke Math. J. vol. 16 (1949) pp. 225-235.

2. M. K. Fort, Jr., A proof that the group of all homeomorphisms of the plane onto itself is locally arcwise connected, Proc. Amer. Math. Soc. vol. 1 (1950) pp. 59-62.

3. J. W. T. Youngs, The topological theory of Frechet surfaces, Ann. of Math. vol. 45 (1944) pp. 753-785.

The UNIVERSITY OF VIRGINIA AND The University of Georgia 